Research Article

\title{
Hybrid Censoring Schemes with Mixture Gamma Failure Distribution
}

\author{
Hassan M. Aljohani $(D)$ and Nada M. Alfaer \\ Department of Mathematics \& Statistics, Faculty of Science, Taif University, Taif 21944, Saudi Arabia \\ Correspondence should be addressed to Hassan M. Aljohani; h.m.sarhan@gmail.com
}

Received 5 November 2020; Revised 4 February 2021; Accepted 23 March 2021; Published 5 April 2021

Academic Editor: Hijaz Ahmad

Copyright ( $) 2021$ Hassan M. Aljohani and Nada M. Alfaer. This is an open access article distributed under the Creative Commons Attribution License, which permits unrestricted use, distribution, and reproduction in any medium, provided the original work is properly cited.

\begin{abstract}
Censoring schemes have received much attention over the past decades. Hybrid censoring schemes are censoring schemes mixed of type-I (T-1) and type-II (T-2) censoring schemes, a most popular area of study in life-testing or reliability experiments. More precisely, hybrid censoring can be described as a mixture of T-I and T-2 schemes. Gamma distribution is widely used, and its connection has more distributions. Mixture and single gamma distribution will be studied to estimate parameters, based on typeII hybrid censoring schemes (T-2HCS). We will apply algorithms to compute the maximum likelihood (ML) estimators and Bayesian approaches, using statistics, such as Markov chain Monte Carlo methods. Bayes estimators and corresponding highest posterior density confidence intervals will be tabled. Also, Markov chain Monte Carlo simulation is implemented to compare the performances of the different methods and the real dataset is analyzed for illustrative purposes.
\end{abstract}

\section{Introduction}

Over the last century, monitoring items and products are incredibly important tasks for managers in companies and different institutions. Censoring scheme data can be a result of monitoring. These kinds of data have received huge attention in recent literature. The main reason for studying this type of data is to understand the underlying mechanisms and then provide useful information for prediction. There are two types of censoring schemes. The first is called T-1, while the other is $\mathrm{T}-2$, which are applied in many scientific applications. More precisely, suppose that a number of units are examined, say $n$. In the case of T-1 censoring schemes, the experiment will be stopped when a predetermined time of the experiment has been reached, say $T$, and all items are failed after that time is not spotted. While T-2 censoring schemes, the experiment is stopped when a predetermined number is chosen, say $r$, from a number of $n$ items, that has failed. Hence, the assumption is not to replace the number of failed items. So, there are two random variables $r$ and $T$ in these types of censoring schemes, see Leese and Patel and Gajjar $[1,2]$ for an explicit motivation. In contrast, the
T-2HCS can be explained as a mixture of two kinds of censoring schemes. The first is T-1 censoring scheme, and the second is T-2 censoring scheme. Suppose that a number of identical units, say $n$, are tested. The test will be finished when a preselected number, say $r$, from a number of items that are failed, or when a predetermined time, say, $T$, on the test has been reached. This type of technique was first introduced by Epstein [3]. Several authors have studied T-2HCS. For example, Bartholomew [4] made a statistical theory about the mean of an exponential distribution, where the sample is chosen from T-2HCS, while Barlow and Madansky [5] solved estimation using a truncated or censored sample. Exact point and interval estimation for an exponential parameter using T-2HCS was studied by Chen and Bhattacharyya [6]. The T-2HCS was also studied under accelerated life tests from Gompertz distribution by Almarashi and Abd-Elmougod [7]. The gamma distribution was studied to derive integral representations for the first and the second type of Chebyshev polynomials by Cesarano [8], who used the fundamentals of Hermite polynomials to find the integration of gamma distribution. Double gamma probability density function was studied [9], which can be 
used with wavelet basis, such Harr wavelet, to reduce spark from recoding of magnetic readings in single-fiber measurement procedure, see [10] for more details. This process is to understand the relationship between spectrum and the results of magnetic readings of features of nanofibers. It is easy to find the explanation of the mixing of censoring schemes. For example, Childs et al. [11] suggested a new approach to the schemes and is called T-2HCS. The definition of data is to suppose $n$ identical products or items on the experiment, and then the experiment will be stopped at the random time, say $T^{*}=\max \left\{x_{r: n}, T\right\}$, where $0 \leq r \leq n$ and $T$ are chosen numbers and $x_{r: n}$ indicates the time of $r$-th failure item in a sample of size $n$. Under this definition, it is clear to extricate three cases:

(1) Case I can be explained as collecting $r$-th failure of items before the moment of $T_{1}$ is reached, and then the experiment can be terminated at $T_{1}$

(2) Case II is to collect $r$-th failure of products, which occurs between two prefixed times, and then the experiment terminated at the last failure item $x_{r: n}$

(3) Case III is to collect the $r$-th failure occurring before time $T_{2}$ is reached

This type of investigation might arise in a case when the examiner decides to add a specific condition Childs et al. [11], for example, at least number of failures $r$ must be collected and then this might cost for the use of the testing facility until the moment of units time $T_{1}$. If the $r$-th failure products occur early, then the examiner can continue until the moment of unit time $T_{1}$ happened to adjust the use of the testing facility. On the other hand, the examiner will surely choose to continue until $r$-th failure of items is collected, see Childs et al. [11] for more details. Several results for the T-2HCS can be found by Kundu and Pradhan [12] who studied the estimation of the parameters of the generalized exponential distribution, and Dube et al. [13] who studied the log-normal distribution using T-2HCS. The main ideas behind the theory of gamma distribution can be found in the early eighteenth century. It was first introduced by Leonhard Euler and Christian Goldbach in 1729 [14]. However, the distribution of gamma has two parameters, which appeared to be more popular in econometrics and certain other scientific areas of study. For example, it is often used to model waiting times, such as life testing. Also, the gamma distribution is more common in the Bayesian approach, which is applied as a conjugate prior distribution for various types of inverse scale parameters such as the variance of noise, see [15-17] for more details. More recently, gamma distribution was generalized by Stacy [18]. Also, Suksaengrakcharoen and Bodhisuwan [19] presented a new distribution, which is called the mixed generalized gamma distribution. The huge problem of applying the Bayes theorem is detecting the posterior distribution, which is more complicated for an analytical solution. The MCMC tools have been involved in many published articles in the past century. Scientists have increasingly applied the approach to drawing a sample from complex and complicated posterior Chib and Greenberg [20]. The use of these tools dates back to the early 70 years
[21] and was generalised and extended by Hastings [22]. The designs of the MCMC approaches were improved by Geman and Geman [23], who added a new tool, simulated annealing steps, which is a stochastic optimization rule. Recently, Julian et al [24] gave a good review of the use of MCMC algorithms. Also, the convergence monitoring process is more effectively improved by Gelman and Rubin [25] in different ways. More information on this process can be found by Aykroyd [26]. Within this article, the investigation of T-2HCS is considered. The project will be based on the background of the gamma distribution. In Section 3, the likelihood function is considered, and also survival function, failure-rate function, and coefficient of variation are investigated. In Section 4, Bayesian approaches are used to introduce a model. Section 5 gives the application of real data which has been analyzed, and the MCMC algorithms for estimating the underlying parameters and its interval estimation is obtained. Section 6 displays conclusions and discussion.

\section{Gamma Distribution}

Suppose that the lifetime $X$ has a gamma distribution with two parameters $\alpha$ and $\beta$ that are the shape and the scale, respectively. Thus, $X \sim G(\alpha,(1 / \beta))$, with density

$$
f(x ; \alpha, \beta)=\frac{1}{\Gamma(\alpha) \beta^{\alpha}} x^{\alpha-1} \exp \left\{-\frac{x}{\beta}\right\}, \quad x>0 ;(\alpha, \beta)>0,
$$

where the exception $\alpha \beta$, the variance $\alpha \beta^{2}$, and $\{X(t), t>0\}$. The cumulative distribution function (CDF), survival function $R(t)$, and failure rate function $H(t)$ are given by

$$
\begin{aligned}
F(x ; \alpha, \beta) & =\int_{0}^{x} \frac{1}{\Gamma(\alpha) \beta^{\alpha}} u^{\alpha-1} \exp \left\{-\frac{u}{\beta}\right\} \mathrm{d} u \\
& =1-s(x ; \alpha, \beta) \exp \left\{-\frac{x}{\beta}\right\}, \quad x>0 ; \beta>0, \alpha \geq 1,
\end{aligned}
$$

where the part $s(x ; \alpha, \beta)=\sum_{j=0}^{\alpha-1}(1 / j !)(x / \beta)^{j}$ and

$$
\begin{aligned}
& R(t \mid \alpha, \beta)=s(x ; \alpha, \beta) \exp \left\{-\frac{t}{\beta}\right\}, \quad t>0 ; \beta>0, \alpha \geq 1, \\
& H(t \mid \alpha, \beta)=\frac{t^{\alpha-1}}{\Gamma(\alpha) \beta^{\alpha} s(x ; \alpha, \beta)}, \quad t>0 ; \beta>0, \alpha \geq 1,
\end{aligned}
$$

where $\alpha \geq 1, \beta>0$, and $0<\omega \leq 1$. The coefficient of variation $(\mathrm{CV})$ of the distribution $G(\alpha,(1 / \beta))$ is defined as

$$
\text { C. } \mathrm{V}=\frac{1}{\sqrt{\alpha}} .
$$

The random variable $X$ of lifetime is said to have a mixture of gamma distribution, if it has the following CDF for each element in the vector $\Omega_{n}=\left\{\omega_{1}, \omega_{2}, \ldots, \omega_{n}, \alpha_{1}\right.$, $\left.\alpha_{2}, \ldots, \alpha_{n}, \beta_{1}, \beta_{2}, \ldots, \beta_{n}\right\}$ greater than zero and $x>0$ as follows: 


$$
f\left(x ; \Omega_{n}\right)=\sum_{i=1}^{n} \omega_{i} f\left(x ; \alpha_{i}, \beta_{i}\right), \quad x>0 ;\left(\alpha_{i}, \beta_{i}\right)>0,0<\omega \leq 1 .
$$

(6)
If the vector $\Omega_{2}=\left\{\omega_{1}, \omega_{2}, \alpha_{1}, \alpha_{2}, \beta_{1}, \beta_{2}\right\}$, then the following CDF is given by

$$
f\left(x ; \Omega_{2}\right)=\frac{\omega_{1}}{\Gamma\left(\alpha_{1}\right) \beta_{1}^{\alpha_{1}}} x^{\alpha_{1}-1} \exp \left\{-\frac{x}{\beta_{1}}\right\}+\frac{\left(1-\omega_{1}\right)}{\Gamma\left(\alpha_{2}\right) \beta_{2}^{\alpha_{2}}} x^{\alpha_{2}-1} \exp \left\{-\frac{x}{\beta_{2}}\right\}, \quad x>0 ;\left(\alpha_{1}, \beta_{1}, \alpha_{2}, \beta_{2}\right)>0,
$$

where $\omega_{2}=1-\omega_{1}$. The corresponding cumulative function (CDF), survival function $R(t)$, and failure-rate function $H(t)$ are given by

$$
\begin{gathered}
F\left(x ; \Omega_{2}\right)=2-\omega_{1} s\left(x ; \alpha_{1}, \beta_{1}\right) \exp \left\{-\frac{x}{\beta_{1}}\right\}-\left(1-\omega_{1}\right) s\left(x ; \alpha_{2}, \beta_{2}\right) \exp \left\{-\frac{x}{\beta_{2}}\right\}, \\
R\left(t \mid \omega_{1}, \alpha_{1}, \beta_{1}, \alpha_{2}, \beta_{2}\right)=\omega_{1} s\left(x ; \alpha_{1}, \beta_{1}\right) \exp \left\{-\frac{t}{\beta_{1}}\right\}+\left(1-\omega_{1}\right) s\left(x ; \alpha_{2}, \beta_{2}\right) \exp \left\{-\frac{t}{\beta_{2}}\right\}-1, \\
H\left(t \mid \omega_{1}, \alpha_{1}, \beta_{1}, \alpha_{2}, \beta_{2}\right)=\frac{\left(\omega_{1} /\left(\Gamma\left(\alpha_{1}\right) \beta_{1}^{\alpha_{1}}\right)\right) x^{\alpha_{1}-1} \exp \left\{-\left(x / \beta_{1}\right)\right\}+\left(\left(1-\omega_{1}\right) / \Gamma\left(\alpha_{2}\right) \beta_{2}^{\alpha_{2}}\right) x^{\alpha_{2}-1} \exp \left\{-\left(x / \beta_{2}\right)\right\}}{\omega_{1} s\left(x ; \alpha_{1}, \beta_{1}\right) \exp \left\{-\left(t / \beta_{1}\right)\right\}+\left(1-\omega_{1}\right) s\left(x ; \alpha_{2}, \beta_{2}\right) \exp \left\{-\left(t / \beta_{2}\right)\right\}-1},
\end{gathered}
$$

where $\left(\alpha_{1}, \alpha_{2}\right) \geq 1,\left(\beta_{1}, \beta_{2}\right)>0$, and $0<\omega_{1} \leq 1$. The coefficient of variation $(\mathrm{CV})$ is given by

$$
\mathrm{C} . \mathrm{V}=\frac{\omega_{1} \beta_{1} \sqrt{\alpha_{1}}+\left(1-\omega_{1}\right) \beta_{2} \sqrt{\alpha_{2}}}{\alpha \beta} .
$$

Figure 1 displays an example single gamma distribution with parameters $\alpha=2$ and $\beta=2$ (a), and mixed gamma distribution with $\alpha=(20,13), \beta=(10,15)$ with $\omega=4$ (b).

The previous forms will be used for the estimation in Section 3, where hybrid censoring schemes requires $f\left(x ; \Omega_{2}\right)$ and $F\left(x ; \Omega_{2}\right)$ in $(7)$ and $(8)$. These forms are used usually to build the likelihood.

\section{Likelihood Function}

At the start of this section, the likelihood function and the maximum likelihood of parameters $\alpha$ and $\beta$ are showed. The likelihood function uses the sample of T-2HCS $\mathbf{x}$ is

$$
L(\alpha, \beta \mid \mathbf{x})=\frac{n !}{\left(n-s_{0}\right) !} \prod_{i=1}^{s_{0}} f\left(x_{i}\right)\left\{1-F\left(t_{0}\right)\right\}^{n-s_{0}},
$$

where

$$
T-2 \operatorname{HCS}_{\left(s_{0}, t_{0}\right)}= \begin{cases}\left(R, x_{R: n}\right), & \text { for the case } 1, \text { where } x_{R: n}>T_{1}, \\ \left(n_{1}, T_{1}\right), & \text { for the case } 2, \text { where } n_{1}>R \\ \left(n, x_{n: n}\right), & \text { for the case } 3, \text { where } T_{2}>x_{n: n} .\end{cases}
$$

Hence, $R$ refers to the number of failures in sample and $\sum_{i=1}^{r} R_{i}<n$. Using (1) and (2) in (12) and if the vector $\Omega_{1}=\left\{\alpha_{1}, \alpha_{2}, \beta_{1}, \beta_{2}\right\}$, then we get the likelihood function of $(\alpha, \beta)$ under the generalized T-2HCS is

$$
L\left(\Omega_{1} \mid \mathbf{x}\right)=\frac{n !}{\left(n-s_{0}\right) !}\left(\frac{1}{\Gamma(\alpha) \beta^{\alpha}}\right)^{s_{0}} \prod_{i=1}^{s_{0}} x_{i}^{\alpha-1} \exp \left\{-\frac{x_{i}}{\beta}\right\}\left\{\sum_{j=0}^{\alpha-1} \frac{1}{j !}\left(\frac{t_{0}}{\beta}\right)^{j} \exp \left\{-\frac{t_{0}}{\beta}\right\}\right\}^{n-s_{0}} .
$$

The log-likelihood function is given by

$$
\ell\left(\Omega_{1}|\mathbf{x}|\right)=\log \left(\frac{n !}{\left(n-s_{0}\right) !}\right)-s_{0} \alpha \log \beta-s_{0} \log \Gamma(\alpha)+(\alpha-1) \sum_{i=1}^{s_{0}} \log x_{(i)}-\sum_{i=1}^{s_{0}} \frac{x_{i}}{\beta}+\left(n-s_{0}\right)\left(\log \sum_{j=0}^{\alpha-1} \frac{1}{j !}\left(\frac{t_{0}}{\beta}\right)^{j}-\frac{t_{0}}{\beta}\right) .
$$




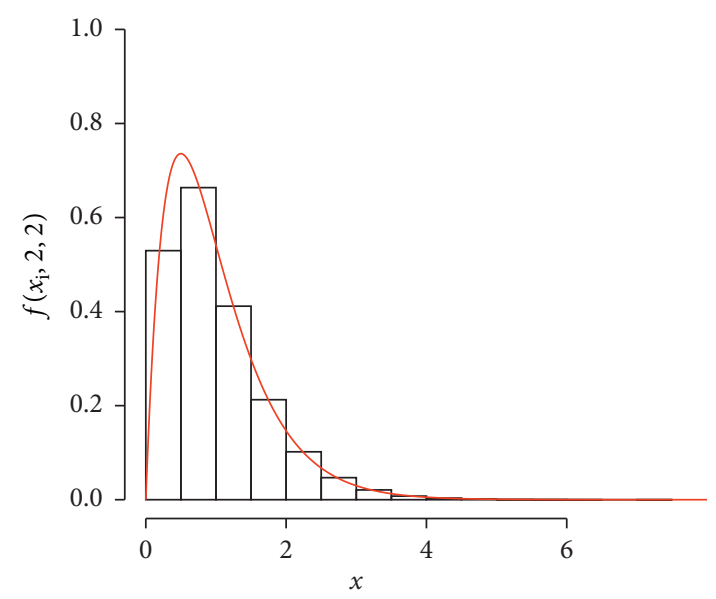

(a)

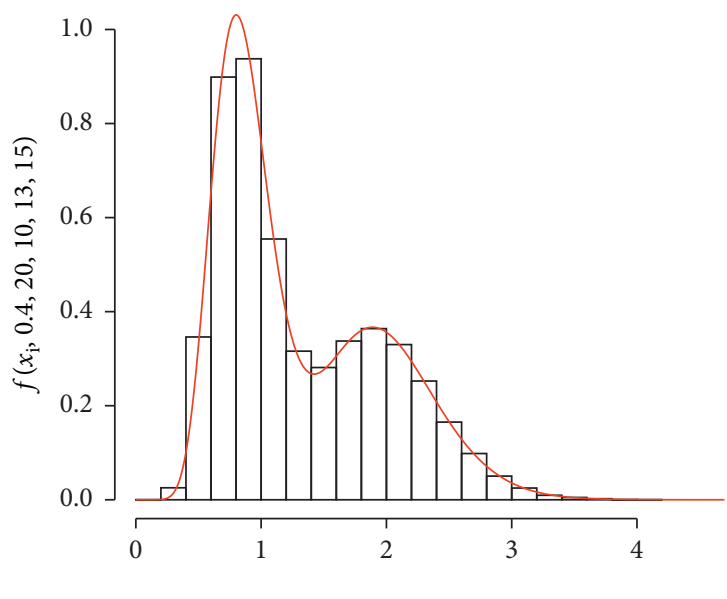

(b)

FIGURE 1: Examples of the scaled gamma distribution: (a) single distribution and (b) mixture of two distributions.

The ML estimate of $\alpha$ and $\beta$ can be obtained by solving the following equations:

$$
\begin{array}{r}
s_{0} \log \beta-s_{0} \frac{(\partial / \partial \alpha) \Gamma(\alpha)}{\Gamma(\alpha)}+\sum_{i=1}^{s_{0}} \log x_{(i)}+\left(n-s_{0}\right)\left(\frac{1}{\alpha-1}+\frac{\ln \left(t_{0} / \beta\right)\left(t_{0} / \beta\right)^{\alpha-1}}{\left(t_{0} / \beta\right)^{\alpha-1}}\right)=0, \\
s_{0} \alpha \frac{1}{\beta}+\sum_{i=1}^{s_{0}} \frac{x_{(i)}}{\beta^{2}}+\left(n-s_{0}\right)\left(\frac{t_{0}}{\beta^{2}}-\frac{\sum_{j=1}^{\alpha-1}(1 /(j-1) !)\left(t_{0} / \beta^{2}\right)^{j-2}}{\sum_{j=0}^{\alpha-1}(1 / j !)\left(t_{0} / \beta\right)^{j}}\right)=0 .
\end{array}
$$

It can be seen that it is not easy to solve the above equations and it can be solved using the iterative method. There are many ways to compute $\widehat{\alpha}$ and $\widehat{\beta}$, one of these is ML estimation to compute $\widehat{\alpha}$ and $\widehat{\beta}$, which can be described as follows:

(i) Starting with an initial value of $\alpha$ and $\beta$, let $\alpha^{(0)}$ and $\beta^{(0)}$, then compute $\ell\left(\mathbf{x} \mid \Omega_{1}\right)^{(0)}$ in (14)

(ii) Generate $\epsilon_{1}$ from a normal distribution, and generate $\epsilon_{2}$ from a normal distribution as well

(iii) Let $\alpha^{(*)}=\alpha^{(i-1)}+\varepsilon_{1}$ and $\beta^{(*)}=\beta^{(i-1)}+\varepsilon_{2}$, then compute $\ell\left(\mathbf{x} \mid \Omega_{1}\right)^{(*)}$

(iv) If $\ell\left(\mathbf{x} \mid \Omega_{1}\right)^{(*)}-\ell\left(\mathbf{x} \mid \Omega_{1}\right)^{(i-1)} \geq \log U(0,1)$, let $\alpha^{(i)}=$ $\alpha^{(*)}$ and $\beta^{(i)}=\beta^{(*)}$; otherwise, $\alpha^{(i)}=\alpha^{(i-1)}$ and $\beta^{(i)}=\beta^{(i-1)}$

(v) Update $\varepsilon_{1}$ and $\varepsilon_{2}$

Using simulated ML samples with the following equation:

Let $\zeta=(\alpha, \beta, R(t), H(t), C V)$, then

$$
\begin{aligned}
\widehat{\zeta}_{j} & =\frac{1}{N-M-1} \sum_{i=M+1}^{N} \zeta_{i}^{(i)}, \\
\operatorname{Var}\left(\widehat{\zeta}_{j}\right) & =\frac{1}{N-M-2} \sum_{i=M+1}^{N}\left(\hat{\zeta}_{j}^{(i)}-\widehat{\zeta}_{j}\right)^{2} .
\end{aligned}
$$

The 95\% credible interval, $\left(\widehat{\zeta}_{i} \pm Z_{(\gamma / 2)} \sqrt{\operatorname{Var}\left(\widehat{\zeta}_{i}\right)}\right)$, where $Z$ is the standard normal variate and $\gamma$ is the acceptance ratio. Hence, the main reason for updating $\epsilon_{1}$ and $\epsilon_{2}$ is to obtain the answers more quickly. In this topic, the missing value method of Louis [27] can be mentioned to obtain the asymptotic confidence intervals. Hence, the complete information contains missing information, and this can be written as

$$
I_{X}(x \mid \theta, \beta)=I_{W}(x \mid \theta, \beta)-I_{W \mid x}(x \mid \theta, \beta),
$$

where $I_{X}\left(x \mid \Omega_{i}\right)$ and $i=1,2$ indicate the observation, $I_{W}\left(x \mid \Omega_{i}\right)$ is the complete information, and $I_{W \mid x}\left(x \mid \Omega_{i}\right)$ is the missing information. The Fisher information $I_{X}\left(x \mid \Omega_{i}\right)$ is given by 


$$
I_{X}\left(x \mid \Omega_{i}\right)=E\left(-\frac{\partial^{2} \ell\left(x \mid \Omega_{i}\right)}{\partial \Omega_{i}^{2}} \mid x \in R\right)-\operatorname{Var}\left(\frac{\partial \ell\left(x \mid \Omega_{i}\right)}{\partial \Omega_{i}^{2}} \mid x \in R\right)
$$

and to derive the risk of $I_{X}\left(\Omega_{1} \mid \mathbf{x}\right)$, the form of excepted information and variance was used:

$$
\begin{aligned}
R\left(I_{X}\left(x \mid \Omega_{i}\right)\right)= & \left(E\left(-\frac{\partial^{2} \ell\left(x \mid \Omega_{i}\right)}{\partial \Omega_{i}^{2}} \mid x \in R\right)-I_{W}\left(x \mid \Omega_{i}\right)\right) \\
& +\operatorname{Var}\left(\frac{\partial \ell\left(x \mid \Omega_{i}\right)}{\partial \Omega_{i}^{2}} \mid x \in R\right),
\end{aligned}
$$

where $R\left(I_{X}\left(x \mid \Omega_{i}\right)\right)$ indicate the risk and the estimator $E\left(-\left(\left(\partial^{2} \ell\left(x \mid \Omega_{i}\right)\right) / \partial \Omega_{i}^{2}\right) \mid x \in R\right) \quad$ is biased with bias $E\left(-\left(\left(\partial^{2} \ell\left(x \mid \Omega_{i}\right)\right) / \partial \Omega_{i}^{2}\right) \mid x \in R\right)-I_{W}\left(x \mid \Omega_{i}\right)$, see $[27,28]$ for more details.

If the vector $\Omega_{2}$, then we get the likelihood function of $(\alpha, \beta)$ under the generalized T-2HCS $\mathbf{x}$ :

$$
\begin{aligned}
L\left(\Omega_{2} \mid \mathbf{x}\right)= & \frac{n !}{\left(n-s_{0}\right) !} \prod_{i=1}^{s_{0}}\left(\frac{\omega_{1}}{\Gamma\left(\alpha_{1}\right) \beta_{1}^{\alpha_{1}}} x_{(i)}^{\alpha_{1}-1} \exp \left\{-\frac{x_{(i)}}{\beta_{1}}\right\}+\frac{\left(1-\omega_{1}\right)}{\Gamma\left(\alpha_{2}\right) \beta_{2}^{\alpha_{2}}} x_{(i)}^{\alpha_{2}-1} \exp \left\{-\frac{x_{(i)}}{\beta_{2}}\right\}\right) \\
& \left\{\omega_{1} \sum_{j=0}^{\alpha_{1}-1} \frac{1}{j !}\left(\frac{t_{0}}{\beta_{1}}\right)^{j} \exp \left\{-\frac{t_{0}}{\beta_{1}}\right\}+\left(1-\omega_{1}\right) \sum_{j=0}^{\alpha_{2}-1} \frac{1}{j !}\left(\frac{t_{0}}{\beta_{2}}\right)^{j} \exp \left\{-\frac{t_{0}}{\beta_{2}}\right\}-1\right\},
\end{aligned}
$$

where $\left(\alpha_{1}, \alpha_{2}\right) \geq 1,\left(\beta_{1}, \beta_{2}\right)>0, \quad$ and $\quad 0<\omega_{1} \leq 1$. The log-likelihood equation is

$$
\begin{aligned}
\ell\left(\Omega_{2} \mid \mathbf{x}\right)= & \log \frac{n !}{\left(n-s_{0}\right) !}+\log \sum_{i=1}^{s_{0}}\left(\frac{\omega_{1}}{\Gamma\left(\alpha_{1}\right) \beta_{1}^{\alpha_{1}}} x_{(i)}^{\alpha_{1}-1} \exp \left\{-\frac{x_{(i)}}{\beta_{1}}\right\}+\frac{\left(1-\omega_{1}\right)}{\Gamma\left(\alpha_{2}\right) \beta_{2}^{\alpha_{2}}} x_{(i)}^{\alpha_{2}-1} \exp \left\{-\frac{x_{(i)}}{\beta_{2}}\right\}\right) \\
& +\left(n-s_{0}\right) \log \left(\omega_{1} \sum_{j=0}^{\alpha_{1}-1} \frac{1}{j !}\left(\frac{t_{0}}{\beta_{1}}\right)^{j} \exp \left\{-\frac{t_{0}}{\beta_{1}}\right\}+\left(1-\omega_{1}\right) \sum_{j=0}^{\alpha_{2}-1} \frac{1}{j !}\left(\frac{t_{0}}{\beta_{2}}\right)^{j} \exp \left\{-\frac{t_{0}}{\beta_{2}}\right\}-1\right) .
\end{aligned}
$$

ML estimation will be used to compute $\widehat{\alpha}_{1}, \widehat{\alpha}_{2}, \widehat{\beta}_{1}$, and $\widehat{\beta}_{2}$ :

(i) Starting with an initial guess of $\widehat{\omega}_{1}, \widehat{\alpha}_{1}, \widehat{\alpha}_{2}, \widehat{\beta}_{1}$ and $\widehat{\beta}_{2}$, say $\widehat{\omega}_{1}^{(0)}, \widehat{\alpha}_{1}^{(0)}, \widehat{\alpha}_{2}^{(0)}, \widehat{\beta}_{1}^{(0)}$ and $\widehat{\beta}_{2}^{(0)}$, by using (3) compute then compute $\ell\left(\Omega_{2} \mid \mathbf{x}\right)^{(0)}$ in (23)

(ii) Generate $\varepsilon_{1}$ and $\varepsilon_{2}, \varepsilon_{3}, \varepsilon_{4}$, and $\varepsilon_{5}$ from a normal distribution

(iii) Let $\alpha_{1}^{(*)}=\alpha_{1}^{(i-1)}+\varepsilon_{1}, \quad \alpha_{2}^{(*)}=\alpha_{2}^{(i-1)}+\varepsilon_{2}, \quad \beta_{1}^{(*)}=$ $\beta_{1}^{(i-1)}+\varepsilon_{3}, \beta_{2}^{(*)}=\beta_{2}^{(i-1)}+\varepsilon_{4}$, and $\omega_{1}^{(*)}=\omega_{1}^{(i-1)}+\varepsilon_{5}$, then compute $\ell\left(\Omega_{2} \mid \mathbf{x}\right)^{(*)}$

(iv) If $\ell\left(\Omega_{2} \mid \mathbf{x}\right)^{(*)}-\ell\left(\Omega_{2} \mid \mathbf{x}\right)^{(i-1)} \geq \log U(0,1), \quad \omega_{1}^{(i)}=$ $\omega_{1}^{(*)}, \alpha^{(i)}=\alpha^{(*)}$, and $\beta^{(i)}=\beta^{(*)}$ otherwise $\omega_{1}^{(i)}=$ $\omega_{1}^{(i-1)}, \alpha^{(i)}=\alpha^{(i-1)}$, and $\beta^{(i)}=\beta^{(i-1)}$

(v) Update $\varepsilon_{1}, \varepsilon_{2}, \varepsilon_{2}$, and $\varepsilon_{4}$

Use simulated ML samples with the following equations. Let $\zeta=\left(\omega_{1}, \omega_{2}, \alpha_{1}, \alpha_{2}, \beta_{1}, \beta_{2}, R(t), H(t), \mathrm{CV}\right)$, then

$$
\begin{aligned}
\widehat{\zeta}_{j} & =\frac{1}{N-M-1} \sum_{i=M+1}^{N} \zeta_{i}^{(i)}, \text { and } \operatorname{Var}\left(\widehat{\zeta}_{j}\right) \\
& =\frac{1}{N-M-2} \sum_{i=M+1}^{N}\left(\hat{\zeta}_{j}^{(i)}-\widehat{\zeta}_{j}\right)^{2} .
\end{aligned}
$$

The 95\% credible interval, $\left(\widehat{\zeta}_{i} \pm Z_{(\gamma / 2)} \sqrt{\operatorname{Var}\left(\widehat{\zeta}_{i}\right)}\right)$, where $Z$ is the standard normal distribution and $\gamma$ is the acceptance ratio. The ML function and estimate point and interval will be used in Section 5. Also, the tools of MCMC algorithms are introduced. However, it is easy to provide that ML often does poorly in estimation since the level of noise usually corrupts the data. Posterior techniques have been recommended to improve ML.

\section{Bayes Estimation}

Bayes estimators are proposed to estimate unknown parameters of gamma distribution based on T-2HCS. Bayesian 
tools require prior distributions to describe the unknown parameters. The gamma distribution is chosen as prior for many reasons. Gamma prior has a fixable form. The posterior distribution of $\alpha$ and $\beta$ given the data is

$$
P(\alpha, \beta \mid \mathbf{x})=\frac{L(\mathbf{x} \mid \alpha, \beta) P_{1}(\alpha) P_{2}(\beta)}{\int_{0}^{\infty} \int_{0}^{\infty} L(\mathbf{x} \mid \alpha, \beta) P_{1}(\alpha) P_{2}(\beta) \mathrm{d} \alpha \mathrm{d} \beta} .
$$

Thus, the Bayes estimate of $g(\alpha, \beta)$ is

$$
E(g(\alpha, \beta))=\frac{\int_{0}^{\infty} \int_{0}^{\infty} g(\alpha, \beta) L(\mathbf{x} \mid \alpha, \beta) P_{1}(\alpha) P_{2}(\beta) \mathrm{d} \alpha \mathrm{d} \beta}{\int_{0}^{\infty} \int_{0}^{\infty} L(\mathbf{x} \mid \alpha, \beta) P_{1}(\alpha) P_{2}(\beta) \mathrm{d} \alpha \mathrm{d} \beta}
$$

It is not easy to evaluate the proportion of the two integrals in Equation (26). Because the integral is too complex and complicated, the MCMC method is proposed to gather samples from the complex form in (25) and then compute an approximation to the exact Bayes estimate of $g(\alpha, \beta)$. We assume that the parameters $\alpha$ and $\beta$ are independent, and the prior distributions of $\alpha$ and $\beta$ are

$$
\begin{aligned}
& \pi_{1}\left(\alpha \mid a_{1}, b_{1}\right)=\frac{b_{1}^{a_{1}}}{\Gamma\left(a_{1}\right)} \alpha^{a_{1}-1} \exp \left\{-b_{1} \alpha\right\}, \quad \alpha>0 ;\left(a_{1}, b_{1}\right)>0, \\
& \pi_{2}\left(\beta \mid a_{2}, b_{2}\right)=\frac{b_{2}^{a_{2}}}{\Gamma\left(a_{2}\right)} \beta^{-a_{2}-1} \exp \left\{-\frac{b_{2}}{\beta}\right\}, \quad \beta>0 ;\left(a_{2}, b_{2}\right)>0,
\end{aligned}
$$

where the parameters of the prior distributions $a, b, c$, and $d$ are suggested to be known for the first initial step of the run and chosen to describe the prior behavior about the unknown parameters using the mean and chosen variance. The joint posterior function of $\alpha$ and $\beta$ can be obtained as follows:

$$
\pi(\alpha, \beta \mid \mathbf{x})=\frac{n !}{\left(n-s_{0}\right) !}\left(\frac{b_{1}^{a_{1}} b_{2}^{a_{2}} \alpha^{a_{1}-1} \beta^{-a_{2}-\alpha-1}}{\Gamma(\alpha) \Gamma\left(a_{1}\right) \Gamma\left(a_{2}\right)}\right)^{s_{0}} \times \prod_{i=1}^{s_{0}} x_{i}^{\alpha-1} \exp \left\{-\frac{x_{i}}{\beta}-\frac{b_{2}}{\beta}-b_{1} \alpha\right\}\left\{\sum_{i=0}^{\alpha-1} \frac{1}{i !}\left(\frac{t_{0}}{\beta}\right)^{i} \exp \left\{-\frac{t_{0}}{\beta}\right\}\right\}^{n-s_{0}} .
$$

It is not easy to solve (11). So, the numerical method such as MCMC methods can be used. From (11), the posterior function of $\alpha$ given $\beta$ is

$$
\pi(\alpha \mid \beta, \mathbf{x}) \propto\left(\frac{\alpha^{a_{1}-1} \beta^{-\alpha}}{\Gamma(\alpha)}\right)^{s_{0}} \exp \left\{\alpha \log \left(\sum_{i=1}^{s_{0}} x_{i}-b_{1}\right)+\left(n-s_{0}\right) \log \sum_{i=0}^{\alpha-1} \frac{1}{i !}\left(\frac{t_{0}}{\beta}\right)^{i}\right\}
$$

and the posterior function of $\beta$ given $\alpha$ is

$$
\pi(\beta \mid \alpha, \mathbf{x}) \propto\left(\beta^{-a_{2}-\alpha-1}\right)^{s_{0}} \exp \left\{-\sum_{i=1}^{s_{0}} \frac{x_{i}}{\beta}-\frac{b_{2}}{\beta}+\left(n-s_{0}\right) \log \sum_{i=0}^{\alpha-1} \frac{1}{i !}\left(\frac{t_{0}}{\beta}\right)^{i}-\left\{\frac{t_{0}}{\beta}\right\}^{n-s_{0}}\right\}
$$

The Metropolis-Hastings tool using the normal distribution to generate random samples from (11) is applied. These algorithms are written to estimate parameter in (11), and each step of iteration provides values of $\widehat{\beta}$ and $\widehat{\alpha}$ and then can be used to give $R(t), H(t)$, and the corresponding confidence interval can be investigated. The proposed algorithm of the MCMC method is considered as follows:

(1) Start with guess value $\widehat{\beta}=\beta^{0}, \widehat{\alpha}=\alpha^{0}, a_{1}=a_{1}^{0}$, $a_{2}=a_{2}^{0}, b_{2}=b_{2}^{0}, N=$ number of iteration and $M=$ burn-in

(2) Compute the posterior distribution in (11)

(3) Generate $\alpha^{(*)}=\alpha^{(i-1)}+\varepsilon$ where $\varepsilon \sim N\left(0, \tau_{1}\right)$

(4) Generate $\beta^{(*)}=\beta^{(i-1)}+\varepsilon$ where $\varepsilon \sim N\left(0, \tau_{2}\right)$
(5) Generate $u$ from a uniform distribution, $U(0,1)$, $\alpha^{(i)}=\left\{\alpha^{(*)}, \quad u \leq\left(\left(\pi\left(\alpha^{(*)}, \beta^{(i-1)} \mid \mathbf{x}\right) q\left(\alpha^{(i-1)} \mid \alpha^{(*)}\right)\right) /\right.\right.$ $\left.\left(\pi\left(\alpha^{(i-1)}, \beta^{(i-1)} \mid \mathbf{x}\right) q\left(\alpha^{(*)} \mid \alpha^{(i-1)}\right)\right)\right) \alpha^{(i-1)}$, O.w

(6) Also, generate $u$ from a uniform distribution, $U(0,1), \quad \beta^{(i)}=\left\{\beta^{(*)}, \quad u \leq\left(\left(\pi\left(\alpha^{(i-1)}, \beta^{(*)} \mid \mathbf{x}\right) q\right.\right.\right.$ $\left.\left.\left(\beta^{(i-1)} \mid \beta^{(*)}\right)\right) /\left(\pi\left(\alpha^{(i-1)}, \beta^{(i-1)} \mid \mathbf{x}\right) q\left(\beta^{(*)} \mid \beta^{(i-1)}\right)\right)\right)$ $\beta^{(i-1)}$, O.w

(7) Update $a_{1}, a_{2}, b_{2}, \tau_{1}$, and $\tau_{2}$

(8) Using simulated samples, estimate the parameters as follows:

Let $\zeta=(\alpha, \beta, R(t), H(t), \mathrm{CV})$, then $\widehat{\zeta}_{j}=(1 /(N-$ $M-1)) \sum_{i=M+1}^{N} \zeta_{i}^{(i)}$ and $\operatorname{Var}\left(\widehat{\zeta}_{j}\right)=(1 /(N-M-2))$ 
TABLE 1: The results of the proposed methods for the parameters using a single gamma distribution.

\begin{tabular}{|c|c|c|c|c|c|c|c|c|c|}
\hline \multirow{2}{*}{ No } & \multirow{2}{*}{ Method } & \multicolumn{8}{|c|}{ Estimation of parameters } \\
\hline & & $\widehat{a}_{1}$ & $\widehat{b}_{1}$ & $\widehat{a}_{2}$ & $\widehat{b}_{2}$ & $\widehat{\alpha}$ & $\widehat{\beta}$ & $\ell(\widehat{\Theta})$ & $\mathrm{BIC}$ \\
\hline \multirow{8}{*}{1} & \multirow[b]{2}{*}{ MLE } & - & - & - & - & 13.1078 & 6.6432 & -26147.26 & 52303.31 \\
\hline & & - & - & - & - & $\begin{array}{l}(10.0244, \\
16.1912)\end{array}$ & $\begin{array}{l}(5.9873, \\
7.2991)\end{array}$ & $\begin{array}{c}(-26149.36 \\
-26145.16)\end{array}$ & - \\
\hline & \multirow{2}{*}{$\begin{array}{c}\mathrm{MCMC} \\
\mathrm{PM}\end{array}$} & 0.001 & 0.001 & 0.001 & 0.001 & 13.1334 & 6.6563 & -26132.81 & 52274.42 \\
\hline & & - & - & - & - & $\begin{array}{l}(10.2845, \\
15.9823)\end{array}$ & $\begin{array}{l}(6.0517, \\
7.2608)\end{array}$ & $\begin{array}{c}(-26134.75 \\
-26130.87)\end{array}$ & - \\
\hline & \multirow{2}{*}{$\begin{array}{c}\text { MCMC } \\
\text { PM }\end{array}$} & 1.6662 & 0.1291 & 2.3932 & 8.7366 & 12.9896 & 6.5946 & -26145.05 & 52298.90 \\
\hline & & $\begin{array}{l}(1.6547, \\
1.6777)\end{array}$ & $\begin{array}{c}(0.1290, \\
0.1291)\end{array}$ & $\begin{array}{l}(2.3815, \\
2.4049)\end{array}$ & $\begin{array}{l}(8.5070, \\
8.9661)\end{array}$ & $\begin{array}{l}(10.0904, \\
15.8888)\end{array}$ & $\begin{array}{l}(5.9768, \\
7.2124)\end{array}$ & $\begin{array}{l}(-26147.18 \\
-26142.92)\end{array}$ & - \\
\hline & $\begin{array}{l}\text { MCMC } \\
\text { MAP }\end{array}$ & 0.001 & 0.001 & 0.001 & 0.001 & 12.8326 & 6.5239 & -26133.78 & 52276.36 \\
\hline & $\begin{array}{c}\text { MCMC } \\
\text { MAP }\end{array}$ & 1.6621 & 0.1289 & 2.4409 & 9.5673 & 12.7888 & 6.5041 & -26146.13 & 52301.06 \\
\hline \multirow{8}{*}{2} & \multirow[b]{2}{*}{ MLE } & - & - & - & - & 11.2500 & 5.2846 & -31790.99 & 63591.00 \\
\hline & & - & - & - & - & $\begin{array}{l}\text { (8.7029, } \\
13.7971)\end{array}$ & $\begin{array}{l}(4.7678 \\
5.8014)\end{array}$ & $\begin{array}{c}(-31793.0526 \\
-31788.93)\end{array}$ & - \\
\hline & \multirow{2}{*}{$\begin{array}{c}\text { MCMC } \\
\text { PM }\end{array}$} & 0.001 & 0.001 & 0.001 & 0.001 & \multirow{2}{*}{$\begin{array}{l}11.1797 \\
(8.6348, \\
13.7245)\end{array}$} & \multirow{2}{*}{$\begin{array}{c}5.2587 \\
(4.7423 \\
5.7750)\end{array}$} & \multirow{2}{*}{$\begin{array}{c}-31776.4055 \\
(-31778.4529 \\
-31774.35)\end{array}$} & 63561.83 \\
\hline & & - & - & - & - & & & & - \\
\hline & \multirow{2}{*}{$\begin{array}{c}\text { MCMC } \\
\text { PM }\end{array}$} & 0.9876 & 0.0994 & 2.2148 & 5.6306 & 10.9739 & 5.1753 & -31788.27 & \multirow[t]{2}{*}{63585.57} \\
\hline & & $\begin{array}{c}(0.9842, \\
0.9911)\end{array}$ & $\begin{array}{l}(0.0994, \\
0.0994)\end{array}$ & $\begin{array}{l}(2.2050 \\
2.2247)\end{array}$ & $\begin{array}{c}(5.5498, \\
5.7114)\end{array}$ & $\begin{array}{l}(8.5376, \\
13.4101)\end{array}$ & $\begin{array}{l}(4.6819, \\
5.6687)\end{array}$ & $\begin{array}{l}(-31790.17 \\
-31786.38)\end{array}$ & \\
\hline & $\begin{array}{l}\text { MCMC } \\
\text { MAP }\end{array}$ & 0.001 & 0.001 & 0.001 & 0.001 & 10.8762 & 5.1323 & -31777.42 & 63563.86 \\
\hline & $\begin{array}{c}\text { MCMC } \\
\text { MAP }\end{array}$ & 0.7873 & 0.0887 & 2.2089 & 5.5248 & 10.7834 & 5.0936 & -31789.09 & 63587.20 \\
\hline \multirow{8}{*}{3} & \multirow[b]{2}{*}{ MLE } & - & - & - & - & 10.4446 & 4.7340 & -32351.64 & \multirow[t]{2}{*}{64712.34} \\
\hline & & - & - & - & - & $\begin{array}{r}(8.1702, \\
12.7190)\end{array}$ & $\begin{array}{c}(4.2796, \\
5.1884)\end{array}$ & $\begin{array}{l}(-32353.57 \\
-32349.72)\end{array}$ & \\
\hline & & 0.001 & 0.001 & 0.001 & 0.001 & 10.5547 & 4.7808 & -32336.98 & 64683.01 \\
\hline & $\begin{array}{l}\text { MCMC } \\
\text { PM }\end{array}$ & - & - & - & - & $\begin{array}{l}(8.2484, \\
12.8609)\end{array}$ & $\begin{array}{l}(4.3202, \\
5.2414)\end{array}$ & $\begin{array}{l}(-32339.02 \\
-32334.95)\end{array}$ & - \\
\hline & & 1.0082 & 0.1004 & 2.1501 & 4.4552 & 10.2966 & 4.6778 & -32348.71 & 64706.46 \\
\hline & $\begin{array}{c}\text { MCMC } \\
\text { PM }\end{array}$ & $\begin{array}{l}(1.0044, \\
1.0120)\end{array}$ & $\begin{array}{l}(0.1004, \\
0.1004)\end{array}$ & $\begin{array}{l}(2.1407, \\
2.1594)\end{array}$ & $\begin{array}{l}(4.3717, \\
4.5387)\end{array}$ & $\begin{array}{r}(7.9718, \\
12.6213)\end{array}$ & $\begin{array}{c}(4.2127, \\
5.1429)\end{array}$ & $\begin{array}{l}(-32350.72 \\
-32346.69)\end{array}$ & - \\
\hline & $\begin{array}{c}\text { MCMC } \\
\text { MAP }\end{array}$ & 0.001 & 0.001 & 0.001 & 0.001 & 10.2342 & 4.6512 & -32337.98 & 64685.01 \\
\hline & $\begin{array}{c}\text { MCMC } \\
\text { MAP }\end{array}$ & 1.0826 & 0.1040 & 2.2150 & 5.6332 & 10.1236 & 4.6057 & -32349.98 & 64709.01 \\
\hline
\end{tabular}

$\sum_{i=M+1}^{N}\left(\widehat{\zeta}_{j}^{(i)}-\hat{\bar{\zeta}}_{j}\right)^{2}$, where $j=1,2,3,4,5$. The main reason of updating the variance of the proposal is to converge. This procedure depends on the acceptance rate. Hence, to update the hyperparameters $a_{1}, a_{2}, b_{1}$, and $b_{2}$ can be computed as

$$
a_{1}=\frac{\left(\alpha^{2}\right)^{(i-1)}}{v_{1}}, \text { and } b_{1}=\frac{(\alpha)^{(i-1)}}{v_{1}},
$$

where $(\alpha)^{(i-1)}$ obtained from the previous acceptance and $v_{1}=1$ is used. Similarly, for the hyperparameters, $a_{2}$ and $b_{2}$ can be computed as follows:

$$
a_{2}=\frac{(\alpha)^{(i-1)}}{v_{2}}+2, \quad \text { and } b_{2}=(\alpha)^{(i-1)}\left(a_{2}-1\right)
$$

For maximum a posteriori (MAP) estimates, it is obtained by computing

$$
\widehat{\zeta}_{\mathrm{MAP}}=\arg \max _{\zeta} \widehat{\zeta}^{K}
$$

There is a small change to posterior mean using temperature, $T^{k}$, with $T^{k}=(2 /(\log (1+k)))$; this is the one choice of annealing schedule, see [23] for more detail. The reason for the use of temperature is that it gives a quick good estimation of the parameter. If the vector $\Omega_{2}$ in (22), then the 


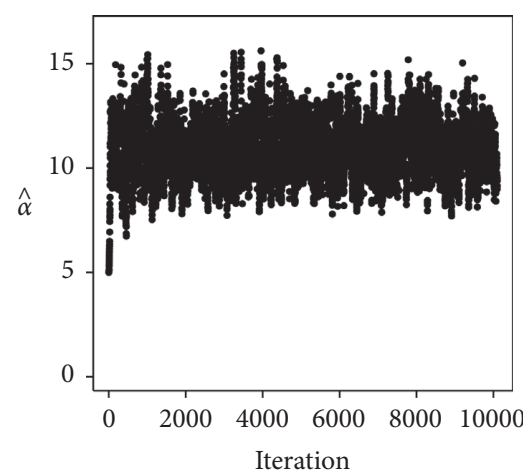

(a)

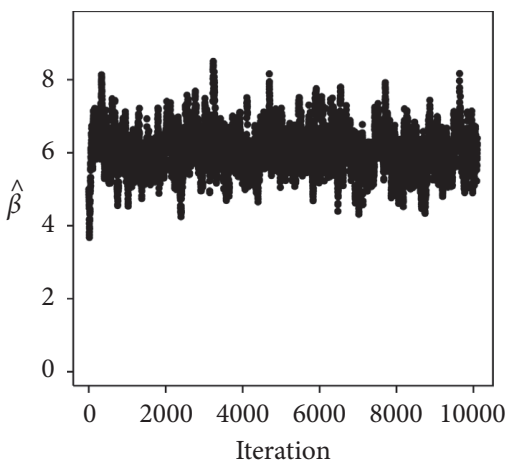

(d)

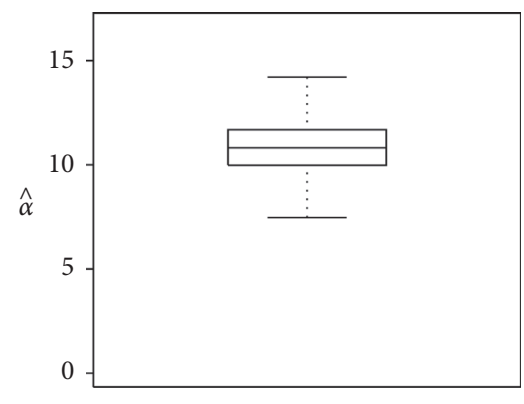

(b)

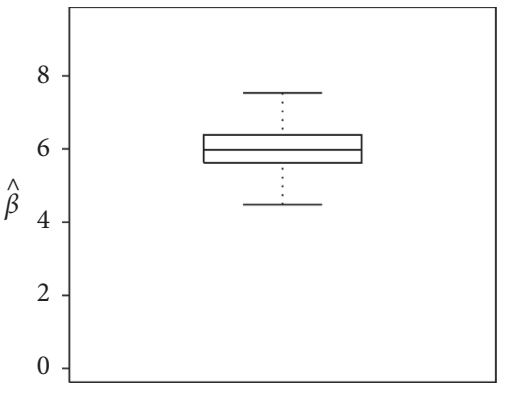

(e)

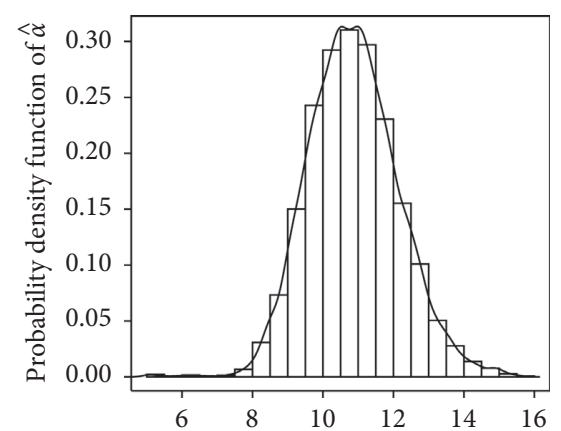

(c)

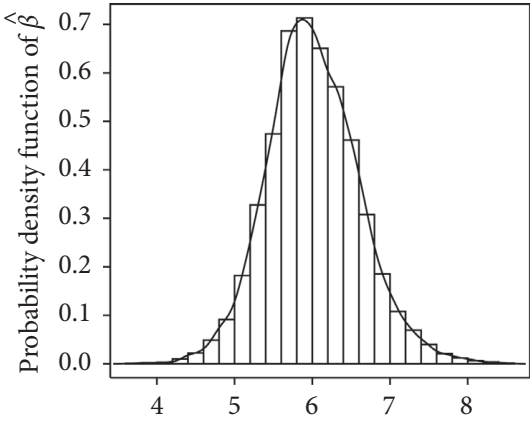

(f)

FIgURE 2: Plots of the monitoring MCMC algorithm where $\alpha$ and $\beta$ are estimated. Scheme 1 at $R=59$ points is used and $t_{0}=2.87$ and $n=60$. (a, d) Trace plots of $\widehat{\alpha}$ and $\widehat{\beta}$ at the transient period of 10000 iterations. (b, c) Summary of the MCMC analysis for $\widehat{\alpha}$ and $\widehat{\beta}$. (e, f) Histograms of $\widehat{\alpha}$ and $\widehat{\beta}$ for scheme 1 .

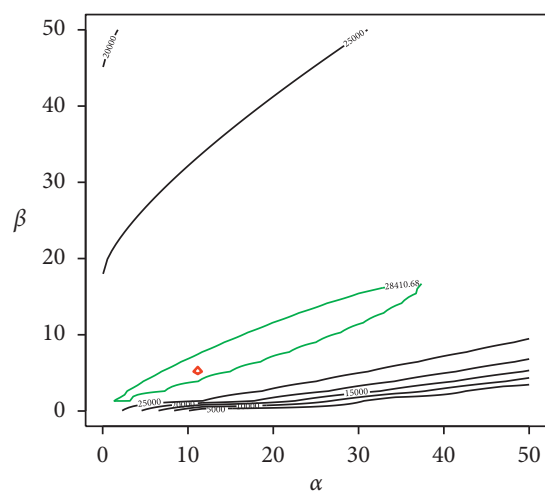

(a)

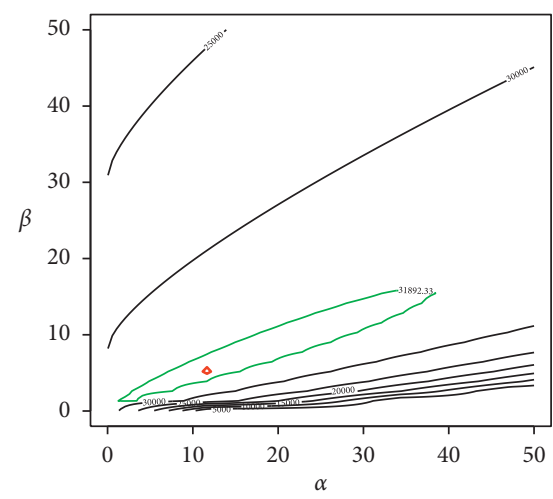

(b)

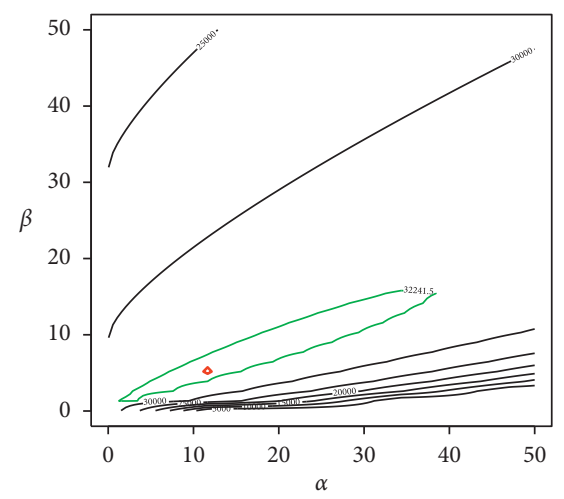

(c)

FIgURE 3: Plots of ML results for two-component models showing the ML estimates (red points) with approximate $95 \%$ confidence fields (gray areas), and the border interval is marked (green lines) for different samples: (a) case 1, (b) case 2, and (c) case 3.

parameter $\omega_{1}$ can be modeled using bate distribution, $\omega \sim$ $\operatorname{Beta}\left(a_{5}, b_{5}\right)$, with density given by

$$
\begin{aligned}
\pi\left(\omega_{1} \mid a_{5}, b_{5}\right) & =\frac{\Gamma\left(a_{5}+b_{5}\right)}{\Gamma\left(a_{5}\right)+\Gamma\left(b_{5}\right)} \omega_{1}^{a_{5}-1}\left(1-\omega_{1}\right)^{b_{5}-1}, \\
0 & \leq \omega \leq 1,\left(a_{5}, b_{5}\right)>0 .
\end{aligned}
$$

Similarly, step $(i-1)$ might provide a mean $\omega_{1}^{(i-1)}$ and variance $V_{5}$ for which correspond to

$$
\begin{aligned}
& a_{5}=\frac{b_{5} \omega_{1}^{(i-1)}}{1-\omega_{1}^{(i-1)}}, \\
& b_{5}=\frac{\left(1-\omega_{1}^{(i-1)}\right)^{2}-v_{5}\left(1-\omega_{1}^{(i-1)}\right)}{v_{5}},
\end{aligned}
$$

where $v_{5}=0.001$ is used. Sometimes, the posterior mean is complex and more complicated. So, the MCMC algorithms 
TABLE 2: The results of the proposed methods for the parameters using the mixed distribution.

\begin{tabular}{|c|c|c|c|c|c|c|c|c|}
\hline \multirow{2}{*}{ No } & \multirow{2}{*}{ Method } & \multicolumn{7}{|c|}{ Estimation of parameters } \\
\hline & & $\widehat{\alpha}_{1}$ & $\widehat{\alpha}_{2}$ & $\widehat{\beta}_{1}$ & $\widehat{\beta}_{2}$ & $\widehat{\omega}$ & $\ell(\widehat{\Theta})$ & $\mathrm{BIC}$ \\
\hline \multirow{5}{*}{1} & & 10.4465 & 7.2604 & 10.8901 & 7.8049 & 0.3713 & -26182.45 & 52382.48 \\
\hline & MLE & $\begin{array}{l}(5.6236 \\
15.2695)\end{array}$ & $\begin{array}{l}(3.8872 \\
10.6336)\end{array}$ & $\begin{array}{l}(8.6102, \\
13.1700)\end{array}$ & $\begin{array}{l}\text { (6.7141, } \\
8.8957)\end{array}$ & $\begin{array}{c}(0.2016 \\
0.5411)\end{array}$ & $\begin{array}{c}(-26187.07 \\
-26177.83)\end{array}$ & - \\
\hline & & 10.3972 & 7.1245 & 12.8093 & 6.5214 & 0.3073 & -26107.13 & 52231.85 \\
\hline & MCMC PM & $\begin{array}{l}(4.5699, \\
16.3643)\end{array}$ & $\begin{array}{l}(6.4403 \\
7.8087)\end{array}$ & $\begin{array}{l}(9.8544 \\
15.7642)\end{array}$ & $\begin{array}{l}(5.2619, \\
7.7810)\end{array}$ & $\begin{array}{l}(0.1106 \\
0.5039)\end{array}$ & $\begin{array}{l}(-26175.48, \\
-26038.79)\end{array}$ & - \\
\hline & $\begin{array}{c}\text { MCMC } \\
\text { MAP }\end{array}$ & 10,0191 & 7.9097 & 10.0580 & 6.4443 & 0.4203 & -26111.28 & 52240.14 \\
\hline \multirow{5}{*}{2} & & 10.9251 & 6.6648 & 9.7085 & 5.7924 & 0.4568 & 31195.37 & 62408.78 \\
\hline & MLE & $\begin{array}{l}(5.1289 \\
16.7213)\end{array}$ & $(4.1246,9.205)$ & $\begin{array}{c}(6.1803 \\
13.2367)\end{array}$ & $\begin{array}{l}(3.6051, \\
7.9796)\end{array}$ & $\begin{array}{c}(0.3845 \\
0.5292)\end{array}$ & $\begin{array}{c}(-46360.62 \\
-16030.12)\end{array}$ & - \\
\hline & & 10.3975 & 5.1517 & 10.9431 & 5.1628 & 0.4903 & -31696.65 & 63411.35 \\
\hline & MCMC PM & $\begin{array}{c}(5.0573, \\
15.7377)\end{array}$ & $\begin{array}{c}(0.2605 \\
10.0430)\end{array}$ & $\begin{array}{c}(8.4918 \\
13.3944)\end{array}$ & $\begin{array}{c}(4.1696 \\
6.1560)\end{array}$ & $\begin{array}{c}(0.4536 \\
0.5269)\end{array}$ & $\begin{array}{l}(-31851.80 \\
-31541.50)\end{array}$ & - \\
\hline & $\begin{array}{c}\text { MCMC } \\
\text { MAP }\end{array}$ & 10.2857 & 4.1613 & 10.9083 & 5.1454 & 0.4919 & -31697.82 & 63413.68 \\
\hline \multirow{5}{*}{3} & & 9.1431 & 5.6311 & 8.4398 & 4.1867 & 0.4101 & -32373.91 & 64765.91 \\
\hline & MLE & $\begin{array}{l}\text { (5.0695, } \\
13.2167)\end{array}$ & $\begin{array}{l}(2.8756 \\
8.3867)\end{array}$ & $(7.0168,9.2768)$ & $\begin{array}{c}(2.5939 \\
5.7795)\end{array}$ & $\begin{array}{c}(0.2930 \\
0.5271)\end{array}$ & $\begin{array}{c}(-32378.93 \\
-32368.88)\end{array}$ & - \\
\hline & & 10.3442 & 4.2784 & 10.4241 & 4.7295 & 0.4303 & -32136.06 & 64290.20 \\
\hline & MCMC PM & $\begin{array}{c}(8.5418, \\
12.1466)\end{array}$ & $(4.1081,4.4486)$ & $\begin{array}{l}\text { (7.9943, } \\
12.8540)\end{array}$ & $\begin{array}{c}(3.7599, \\
5.6990)\end{array}$ & $\begin{array}{c}(0.2333 \\
0.6273)\end{array}$ & $\begin{array}{l}(-32258.16 \\
-32013.95)\end{array}$ & - \\
\hline & $\begin{array}{c}\text { MCMC } \\
\text { MAP }\end{array}$ & 9.2057 & 3.9810 & 10.2048 & 4.6394 & 0.4592 & -32298.07 & 64614.23 \\
\hline
\end{tabular}

are implemented to estimate the parameters using hybrid censoring schemes. In Section 5, the proposed methods will be investigated and compared.

\section{Application to Real-Life Data}

A real application set of carbon fibers is collected by Nichols and Padgett [29]; these data give 100 observations. According to generalized T-2HCS, three different schemes can be obtained from data:

Case 1: if the number of failures is $r=81$, the first time is $T_{1}=3.5$ and the second time of finishing experiments is $T_{2}=4$. Since the last failure is $x_{81: 100}<T_{1}$, the experiment would have terminated in this case at $T_{1}=3.5$, and the sample $0.39,0.81, \ldots, 3.31$ and 3.33 .

Case 2: if the number of item failures is $r=91$, the first time is $T_{1}=3.5$, and the second time is $T_{2}=4$. Since $T_{1}<x_{91: 100}<T_{2}$, the latest failure will be collected is $x_{91: 100}=3.70$ and the sample is $0.39,0.81, \ldots, 3.68$ and 3.70 .

Case 3: if the number of failures is $r=93$, the first time to stop is $T_{1}=3.5$, and the second time to stop is $T_{2}=4$. Since $T_{2}<x_{93: 100}$, the experiment will be finished by $T_{2}=4$ and the sample is $0.39,0.81, \ldots, 3.70$ and 3.75, see [30] for explicit motivation.

The MLEs and Bayes were applied based on the three schemes to estimate $\beta$ and $\alpha$. The confidence intervals are also calculated - the parameter estimation of $\beta$ and $\alpha$ - using the MLEs and Bayes methods. In the single distribution of gamma, the ML and Bayes estimates are applied when the prior parameters are $a_{1}=a_{2}=b_{1}=b_{2}=0.001$ and updated using the forms in (16) and (22). The number of iterations is 10,000 . They were removing the first 1000 values, which is described as burn-in. The Bayesian Information Criterion (BIC) is applied, where it is defined as $p \log (n)-2 \ell(\widehat{\Theta}), p$ is the number of parameters.

Also, consider Table 1. Firstly, two approaches are applied: the first is to fix the hyperparameters and the other is to update the hyperparameters. It can be seen that there is no significant difference between these two methods. In general, $\widehat{\alpha} \sim(10.1236,13.1078)$ and $\widehat{\beta} \sim(4.6057,6.6432)$. According to the BIC, it is clear to identify the posterior mean estimates as the best model.

Figure 2 shows an example of the path of the progress of the MCMC algorithms (PM): (a) and (d) show the values of the proposal for $\alpha$ and $\beta$; (b) and (e) show the boxplot to show the median; (c) and (f) show the density of the estimated parameters.

The contour of ML function is shown in Figure 3, and the summary of the numerical results is shown in Table 1; hence, there is symmetry about the $45^{\circ}$ line. The red dots show the location of the ML, and the dark areas show the $95 \%$ confidence fields for $\alpha$ and $\beta$. The results of estimated parameters $\alpha_{1}, \alpha_{2}, \beta_{1}, \beta_{2}$, and $\omega_{1}$ for the mixed gamma distribution are shown in Table 2 . Hence, $\omega_{2}=1-\omega_{1}$, and the hyperparameters are chosen to be $a_{1}=a_{2}=a_{3}=a_{4}=b_{1}=$ $b_{2}=b_{3}=b_{4}=0.001$ and $a_{5}=b_{5}=2$, where $a_{1}$ and $b_{1}$ are the hyperparameters for $\alpha_{1} ; a_{2}$ and $b_{2}$ are the prior parameters for $\alpha_{2} ; a_{3}$ and $b_{3}$ are the parameters for the distribution of $\beta_{1}$; and $a_{4}$ and $b_{4}$ are the parameters for the distribution of $\beta_{2}$. In 
general, it can be seen that posterior mean gives slightly better results than our Bayesian methods, but the MLEs are simpler to use and much faster and are implemented easily. Finally, we believe mixed models can improve the result of estimation.

\section{Conclusion}

This article has introduced two parameter estimation models that are proposed using MCMC algorithms. Extensive simulation studies have shown that our models work well across a signal and mixture of gamma distribution T-2HCS. The MCMC algorithms were applied to calculate the approximate proposal methods and the corresponding credible intervals. Finally, we summarize some comments about the results shown in Tables 1 and 2 as follows:

(1) The results of proposal methods in Table 2 show that the mixed model is good to use and estimate the parameters, and it is more acceptable

(2) Bayesian approaches give slightly better results than MLEs

(3) Updating the variances of the proposal gives the answer more quickly

(4) The thin number helps to provide the independent sample

(5) The Bayesian information criterion shows that the results improved in case I, when $r$-th failures are collected before reaching the moment of $T_{i}$

(6) Some parameters proposed each iteration, while some of them stop for some steps; this technique helps to converge

(7) Tables show that proposed methods work well for estimating parameters

\section{Data Availability}

The data used to support the findings of this study are included within the article.

\section{Conflicts of Interest}

The authors declare that they have no conflicts of interest.

\section{Acknowledgments}

This study was funded by Taif University Researchers Supporting Project (TURSP-2020/279), Taif University, Taif, Saudi Arabia.

\section{References}

[1] M. N. Leese, "Use of censored data in the estimation of Gumbel distribution parameters for annual maximum flood series," Water Resources Research, vol. 9, no. 6, pp. 1534-1542, 1973.

[2] M. N. Patel and A. V. Gajjar, "Maximum likelihood estimation in compound exponential failure model with changing failure rates from type i progressively censored and group censored samples," Communications in StatisticsTheory and Methods, vol. 21, no. 10, pp. 2899-2908, 1992.

[3] B. Epstein, "Truncated life tests in the exponential case," The Annals of Mathematical Statistics, vol. 25, no. 3, pp. 555-564, 1954.

[4] D. J. Bartholomew, "The sampling distribution of an estimate arising in life testing," Technometrics, vol. 5, no. 3, pp. 361-374, 1963.

[5] R. E. Barlow, A. Madansky, F. Proschan, and E. M. Scheuer, "Statistical estimation procedures for the "burn-in" process," Technometrics, vol. 10, no. 1, pp. 51-62, 1968.

[6] S.-M. Chen and G. K. Bhattacharyya, "Exact confidence bounds for an exponential parameter under hybrid censoring," Communications in Statistics-Theory and Methods, vol. 17, no. 6, pp. 1857-1870, 1988.

[7] A. M. Almarashi and G. A. Abd-Elmougod, "Accelerated competing risks model from gompertz lifetime distributions with type-ii censoring scheme," Thermal Science, vol. 24, no. Suppl. 1, pp. 165-175, 2020.

[8] C. Clemente, "Generalized chebyshev polynomials," Hacettepe Journal of Mathematics and Statistics, vol. 43, no. 5, pp. 731-740, 2014.

[9] E. William Barnes, "The theory of the double gamma function," Philosophical Transactions of the Royal Society of London. Series A, Containing Papers of a Mathematical or Physical Character, vol. 196, no. 274-286, pp. 265-387, 1901.

[10] T. Roodbar Shojaei, A. Hajalilou, M. Tabatabaei, H. Mobli, and M. Aghbashlo, "Characterization and evaluation of nanofiber materials," Handbook of Nanofibers, Springer, Cham, Switzerland, pp. 491-522, 2019.

[11] A. Childs, B. Chandrasekar, N. Balakrishnan, and D. Kundu, "Exact likelihood inference based on type-I and type-II hybrid censored samples from the exponential distribution," Annals of the Institute of Statistical Mathematics, vol. 55, no. 2, pp. 319-330, 2003.

[12] D. Kundu and B. Pradhan, "Estimating the parameters of the generalized exponential distribution in presence of hybrid censoring," Communications in Statistics-Theory and Methods, vol. 38, no. 12, pp. 2030-2041, 2009.

[13] S. Dube, B. Pradhan, and D. Kundu, "Parameter estimation of the hybrid censored log-normal distribution," Journal of Statistical Computation and Simulation, vol. 81, no. 3, pp. 275-287, 2011.

[14] P. J. Davis, "Leonhard euler's integral: a historical profile of the gamma function," The American Mathematical Monthly, vol. 66, no. 10, pp. 849-869, 1959.

[15] D. F. Andrews and C. L. Mallows, "Scale mixtures of normal distributions," Journal of the Royal Statistical Society, Series B, vol. 36, no. 1, pp. 99-102, 1974.

[16] R. V. Hogg and A. T. Craig, "Distribution of functions of random variables," Introduction to Mathematical Statisticspp. 176-179, Macmillan, New York, NY, USA, 4th edition, 1978.

[17] P. Gopalan, J. M. Hofman, and D. M. Blei, "Scalable recommendation with poisson factorization," 2013, http://arxiv. org/abs/1311.1704.

[18] E. W. Stacy, "A generalization of the gamma distribution," The Annals of Mathematical Statistics, vol. 33, no. 3, pp. 11871192, 1962.

[19] S. Suksaengrakcharoen and W. Bodhisuwan, "A new family of generalized gamma distribution and its application," Journal of Mathematics and Statistics, vol. 10, no. 2, p. 211, 2014. 
[20] S. Chib and E. Greenberg, "Understanding the metropolishastings algorithm," The American Statistician, vol. 49, no. 4, pp. 327-335, 1995.

[21] N. Metropolis, A. W. Rosenbluth, M. N. Rosenbluth, A. H. Teller, and E. Teller, "Equation of state calculations by fast computing machines," The Journal of Chemical Physics, vol. 21, no. 6, pp. 1087-1092, 1953.

[22] W. K. Hastings, "Monte Carlo sampling methods using Markov chains and their applications," Biometrika Trust, vol. 57, no. 1, pp. 97-109, 1970.

[23] S. Geman and D. Geman, "Stochastic relaxation, Gibbs distributions, and the Bayesian restoration of images," IEEE Transactions on Pattern Analysis and Machine Intelligence, vol. PAMI-6, no. 6, pp. 721-741, 1984.

[24] B. Julian, P. Green, D. Higdon, and K. Mengersen, "Bayesian computation and stochastic systems," Statistical Science, vol. 10, no. 1, pp. 3-41, 1995.

[25] A. Gelman and D. B. Rubin, "Inference from iterative simulation using multiple sequences," Statistical Science, vol. 7, no. 4, pp. 457-472, 1992.

[26] R. G. Aykroyd, "Statistical image reconstruction," in Industrial Tomography: Systems and Applications, pp. 401-427, Woodhead Publishing, Sawston,United Kingdom, 2015.

[27] T. A. Louis, "Finding the observed information matrix when using the EMAlgorithm," Journal of the Royal Statistical Society: Series B (Methodological), vol. 44, no. 2, pp. 226-233, 1982.

[28] A. Bruce and H.-Ye Gao, "Understanding waveshrink: variance and bias estimation," Biometrika, vol. 83, no. 4, pp. 727-745, 1996.

[29] M. D. Nichols and W. J. Padgett, "A bootstrap control chart for weibull percentiles," Quality and Reliability Engineering International, vol. 22, no. 2, pp. 141-151, 2006.

[30] M. A. W. Mahmoud and M. G. M. Ghazal, "Estimations from the exponentiated Rayleigh distribution based on generalized type-II hybrid censored data," Journal of the Egyptian Mathematical Society, vol. 25, no. 1, pp. 71-78, 2017. 